Musées, Patrimoine et Culture scientifiques et techniques

152 | 2014

mars-avril 2014

\title{
Réflexions sur les stages et les conditions d'accueil d'un stagiaire dans une institution culturelle
}

\section{Serge Chaumier}

\section{OpenEdition \\ Journals}

Édition électronique

URL : http://journals.openedition.org/ocim/1341

DOI : 10.4000/ocim.1341

ISSN : 2108-646X

Éditeur

OCIM

Édition imprimée

Date de publication : 25 mars 2014

ISSN : 0994-1908

Référence électronique

Serge Chaumier, «Réflexions sur les stages et les conditions d'accueil d'un stagiaire dans une institution culturelle », La Lettre de I'OCIM [En ligne], 152 | 2014, mis en ligne le 17 avril 2016, consulté le 19 avril 2019. URL : http://journals.openedition.org/ocim/1341; DOI : 10.4000/ocim.1341 


\title{
Réflexions sur les stages \\ et les conditions d'accueil d'un stagiaire dans une institution culturelle
}

\author{
Serge Chaumier *
}

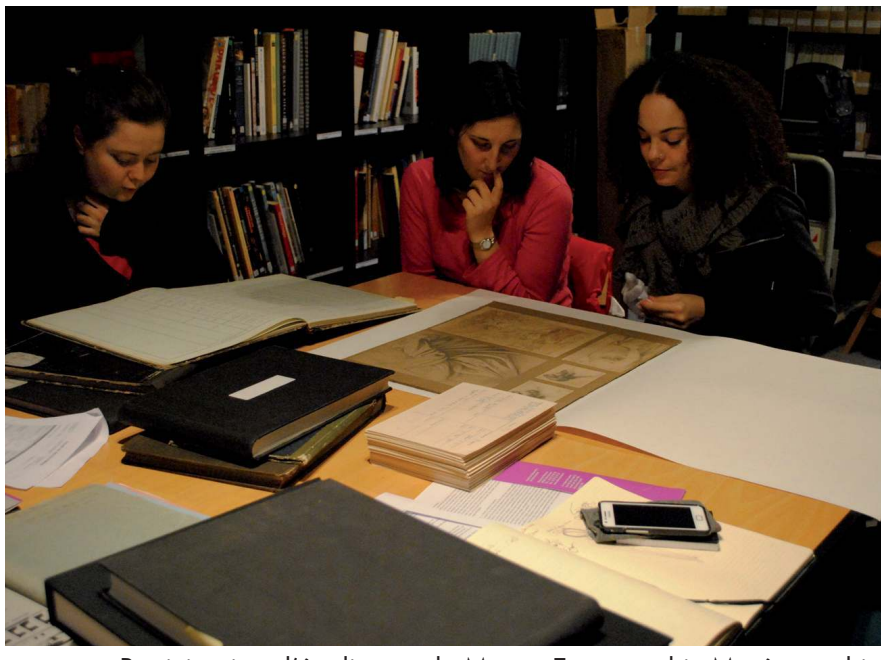

Participation d'étudiantes du Master Expographie-Muséographie (MEM) de l'université d'Artois au travail de récolement au musée des Beaux-Arts de Valenciennes () S. Chaumier
* Serge Chaumier est responsable du Master Expographie-Muséographie à l'université d'Artois serge.chaumier@univ-artois.fr
Partant des nombreuses dérives observées lors des stages, l'auteur propose une sorte de vade-mecum du stage dans les institutions muséales, un répertoire des bonnes pratiques pour faire de cette étape du parcours universitaire un moment privilégié pour expérimenter, se confronter à la réalité du terrain, valider des connaissances en compétences.

La situation des étudiants en recherche de stage dans les entreprises et les institutions culturelles devient désormais complexe et difficile. Si faire un stage durant ses études était quelque chose d'exceptionnel il y a vingt ans, du fait du nombre restreint de formations universitaires professionnalisantes, la situation est plutôt inverse aujourd'hui. Les stages se sont multipliés, le changement du rapport aux études, la sociologie du monde universitaire, des questions internes aux lieux d'accueil, et notamment à leur financement, autant de facteurs pour expliquer ces dérives. Les effets pervers sont nombreux. Autant de raisons pour rappeler les fonctions d'un stage, les rôles dévolus à un stagiaire, les conditions pour qu'un stage soit réussi pour tous, les exigences légitimes envers le stagiaire et son lieu de formation, mais aussi les devoirs et obligations d'une structure d'accueil.

Il s'agit de rappeler des éléments objectifs, mais aussi, en nous appuyant sur une quinzaine d'années d'expériences à l'accompagnement de stages, de partager le fruit de nos réflexions et de nous positionner en affirmant des partis-pris ${ }^{(1)}$. Car nous sommes convaincu que la scientificité ne réside pas dans un faux semblant de neutralité, mais dans la reconnaissance de ses 
subjectivités et dans la mise en évidence des tensions et des contradictions. Les institutions ont tout intérêt à conduire une analyse à ce sujet, sur leurs devoirs et responsabilités, si elles veulent bénéficier d'étudiants motivés et de futurs professionnels engagés et responsables. Quant aux universités, il est très important qu'elles précisent les objectifs et les contenus des stages en entreprise qu'elles prévoient dans le curriculum de formation.

Retenons tout d'abord un seul chiffre : entre 2006 et 2011, soit en l'espace de 5 ans, le nombre de stagiaires en France a été multiplié par deux, comme si l'université était en proie à une véritable « stagiophagie ». Nous sommes ainsi passés de 800000 à 1,5 millions stagiaires par an en $2011^{(2)}$. Certes, tous ne sont pas dans les musées, mais la culture en accueille son lot. Comment expliquer une telle situation, à bien des égards délirante?

\section{Les raisons de la surenchère}

Pour le secteur qui nous préoccupe, nous pouvons identifier trois facteurs majeurs de cette explosion.

D'abord, la montée en puissance depuis trente ans d'un discours sur le rôle de l'université. Celle-ci, qui était dévolue à former aux humanités et à la culture générale pour ensuite laisser les individus s'adapter au monde de l'entreprise, s'est structurellement modifiée. Certains pionniers ont plaidé pour que des formations professionnalisantes soient développées. Rares il y a encore vingt ans, sous la forme des DESS, des IUP, des IUT, le modèle s'est progressivement imposé. Cela a donné lieu à des batailles intestines et fratricides dans les universités, mais bon an mal an, le mot d'ordre a été repris par les ministères successifs et les universitaires ont fini par s'y ranger. Loin d'être une réjouissance, la mutation de l'université s'est réalisée avec une détérioration notable de sa fonction d'origine, mais l'université s'est convertie à la professionnalisation. La réforme dite de Bologne et le passage au LMD a été l'accélérateur du changement. Les licences professionnelles, les masters pro se sont multipliés, au point d'exacerber une concurrence entre universités et diplômes, totalement déconnectée du marché de l'emploi. Devant le succès des formations professionnelles et la déshérence de bien des formations classiques, les universitaires se sont tous convertis, souvent sans grande conviction, à la formation professionnalisante, sans pour autant se donner ni les moyens ni les compétences pour les faire vivre. Le paradoxe est quaujourd'hui se dessine une université largement tournée vers la professionnalisation, mais sans disposer du personnel adéquat pour cela. Car former des étudiants suppose de connaître les métiers auxquels on est sensé préparer, voire

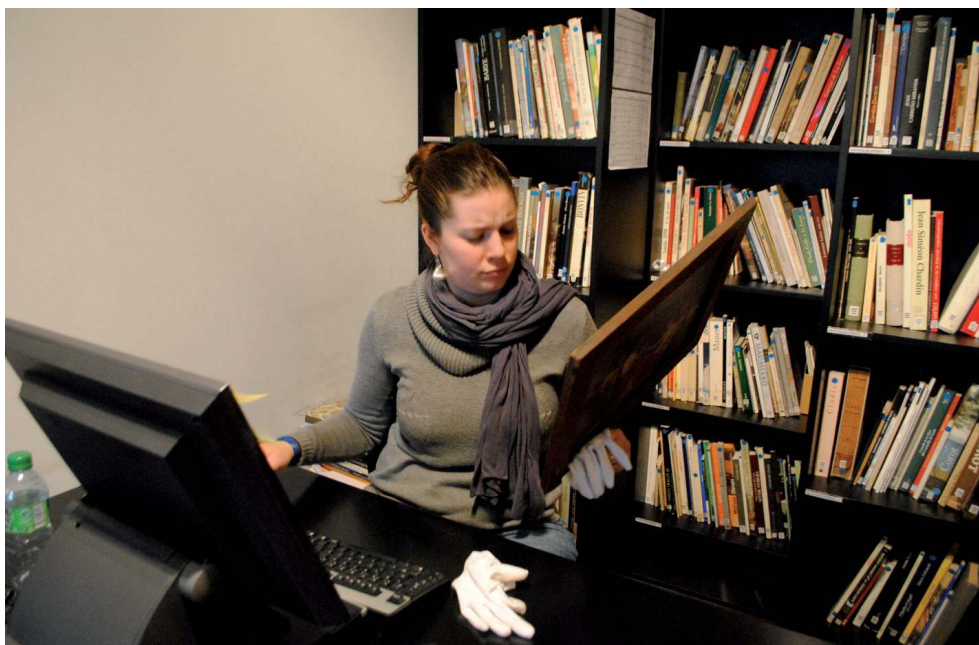

Récolement au musée des Beaux-arts de Valenciennes (C) S. Chaumier

déjà de les identifier avec précision. Dans le meilleur des cas, les formateurs ont su nouer des partenariats pour étudier les réalités du terrain, les besoins et élaborer des référentiels de compétences. Les universitaires ont rarement eu l'occasion de se plonger concrètement dans le milieu en question, et délèguent donc la formation à la vie professionnelle à des intervenants extérieurs (quand il n'y a pas trop de collègues auxquels il faut octroyer un service de cours, ce qui en période de compression budgétaire est une menace évidente). Le fameux stage vient ajouter un gage de crédibilité. Ainsi, ce sont des raisons sociologiques internes à l'université qui expliquent la multiplication des formations, et par conséquent l'exigence de périodes conséquentes de stage ${ }^{(3)}$.

Il en découle une seconde raison : pour qualifier une formation de professionnelle, il s'agit d'y inclure un stage. En effet, si nombre de cursus universitaires se sont convertis à la professionnalisation, il serait erroné de croire qu'ils l'ont fait en s'alignant sur les véritables formations professionnelles, plus gourmandes en moyen. Bien au contraire, ce sont davantage ces dernières qui ont dû se restreindre. Pour le moment de grandes disparités existent, fruit d'une histoire très peu régulée et souvent irrationnelle. Ainsi voit-on des masters s'afficher comme professionnels, avec à peine 200 heures d'encadrement, alors que d'autres plus ambitieux affichent plus de 500 heures. Ce rapport de un à trois n'est évidemment pas le seul critère, la cohérence interne est souvent plus problématique. Trop souvent il s'agit d'agréger les enseignements dont on dispose, au gré des disponibilités de collègues ou d'intervenants croisés ici ou là. La possibilité d'un stage, complété d'interventions de quelques professionnels 
flattés de se voir sollicités pour intervenir dans un cursus universitaire, serviront de caution pour s'affirmer comme formation professionnelle. Évidemment, une formation professionnelle suppose bien autre chose, qui tient plus du compagnonnage ou de l'apprentissage que de la formation académique dans laquelle la plupart des universitaires sont moulés ${ }^{(4)}$. Conduire chacun selon son profil, développer les projets tuteurés collectifs, les expériences sur le terrain, les visites sur site, les coopérations avec le milieu professionnel, les rencontres et les occasions de partage, à chaque fois renouvelées, suppose une présence et une disponibilité que bien peu d'enseignants chercheurs consentent. Pour eux, en effet, la part noble du travail universitaire réside dans la soutenance de thèse, les publications et les colloques. Rappelons que le dispositif pédagogique ne pèse pas lourd, face aux productions d'articles aux yeux de l'AERES, instance chargée d'évaluer les enseignants chercheurs. Bref, les stages sont devenus le seul garant de bien des formations. Parmi cette offre, il ne faut pas non plus oublier toutes les écoles d'enseignement supérieur qui, privées ou non, proposent également leur formation, et donc leurs stagiaires.

Troisième raison, et non des moindres : l'évolution des institutions elles-mêmes, demandeuses de toujours plus de main d'œuvre à bon compte. Car on ne saurait occulter que les restrictions financières qui frappent les grandes comme les petites structures se traduisent souvent par le recrutement de stagiaires à la place de salariés, ou du moins de vacataires auxquels on aurait fait appel auparavant. En oubliant le statut d'un étudiant-stagiaire, sa raison d'être et sa place dans l'entreprise, les demandes les plus contestables sont désormais faites avec le brin de naïveté qui sied à ce qu'un ordre libéral impose désormais comme une évidence dans les têtes. Réduire le coût de la masse salariale passe par le fait de faire appel à des stagiaires, non pour assister le responsable mais parfois pour le remplacer! Ainsi nous avons pu recevoir (et refuser) des offres de stage, où l'on nous demandait d'envoyer un stagiaire tous les 5 mois pour remplacer un poste non pourvu. Ou encore ces structures où l'on demande au stagiaire de former le stagiaire suivant... D'autres estiment qu'avec un bataillon de stagiaires, on maintient la pérennité de la structure, et l'on voit ainsi de grands festivals aux équipes permanentes très réduites maintenir leur activité, mais c'est aussi le cas lorsque les postes de médiateurs sont tous occupés par des stagiaires dans certaines structures d'exposition. Le fait que les étudiants soient mieux formés pour leurs tâches, mais aussi qu'ils soient prêts à ne pas compter leur temps, par passion, mais aussi par jeu concurrentiel, dans un marché de l'emploi saturé, conduit à une surenchère. Et des responsables culturels, pourtant très critiques envers l'ordre économique actuel, sont conduits à reproduire dans leur propre structure des mécanismes similaires. Toujours plus (pour l'employé) pour toujours moins (au niveau financier).

Pour toutes ces raisons, les stages, mais surtout les demandes de stages se multiplient, si bien que les négociations s'opèrent de plus en plus tôt dans l'année, mettant parfois en péril le bon ajustement entre le projet professionnel de l'étudiant, les missions confiées et les besoins de la structure. Un stage efficient répond à une analyse et une réflexion auxquelles doivent s'astreindre l'étudiant, mais aussi le référent universitaire de la formation et le tuteur en entreprise.

\section{L'articulation entre l'offre de stage et la formation}

Même si cela pourra paraître des truismes à certains, rappelons malgré tout à quoi sert un stage et la place qu'il est sensé occuper dans une formation, nous pourrons en déduire les conditions pour que celui-ci soit véritablement opérationnel.

Le stage n'est pas une sorte de cerise sur le gâteau ou de décoration dans un parcours de formation. Il est l'occasion d'expérimenter, de se confronter au réel d'une situation professionnelle et de transformer ses connaissances en compétences. Une formation universitaire professionnelle, par ses apports en enseignements n'apporte pas de compétences, mais des aptitudes que l'expérience seule va pouvoir mettre en œuvre. Ainsi, le stage est un élément fondamental d'un parcours. Il doit être inséré de manière judicieuse et permettre un aller-retour entre mise en pratique et distanciation réflexive. L'idéal est qu'il soit inséré au sein même du cursus, et non à la fin de celui-ci, même si l'on prête souvent au rapport de stage ce moment de retour sur expérience. Encore faut-il que celui-ci soit ensuite discuté et validé avec les responsables dans la formation.

Première conséquence de ce qui précède, le stage, et donc ses missions, doit être en rapport avec ce qui est dispensé dans la formation. Ceci peut sembler une évidence, mais c'est pourtant loin d'être toujours le cas. Ainsi voit-on, par exemple, des missions de stage axées sur l'assistance à la réalisation d'une exposition confiées à un étudiant en étude patrimoine qui ne dispose dans son cursus d'aucun apport préalable dans ce domaine. Si les responsables de la formation doivent être attentifs à l'adéquation entre les missions de stage et le cursus, c'est aussi aux structures d'accueil en entreprise d'y veiller. Devant les quantités pléthoriques de demandes de stage, et devant l'anarchie des formations, les lieux d'accueil prennent parfois des stagiaires

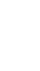

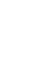

.


venant de n'importe quelle formation sur n'importe quel poste. Ainsi, un poste en communication ne devrait pas faire appel aux mêmes promotions d'étudiants qu'un poste en médiation ou en inventaire du patrimoine bâti. Il y a des formations spécialisées, et l'on ne confie pas une étude des publics à un étudiant qui n'a jamais eu la moindre notion de sociologie. Insistons sur le fait qu'il est aussi de la responsabilité des structures d'accueil à être attentives au recrutement effectué.

Si le stage est véritablement pensé dans son articulation à la formation, il doit être véritablement considéré par celle-ci. C'est une occasion pour produire des allers-retours et de nourrir les expériences pédagogiques par des exemples parlants pour les étudiants. Il est dès lors étonnant de voir des plannings de formations universitaires qui n'y consacrent aucune période en particulier dans l'année, les étudiants choisissant de ne pas aller en cours quand ils sont en stage! Si le stage est primordial, les enseignements ne sont pas des compléments accessoires, les deux doivent s'équilibrer et si possible s'articuler. Ce qui veut dire que le lieu de stage devrait s'intéresser à la formation dispensée et en tous les cas, ne pas demander à ce que le stagiaire soit présent à plein temps toute l'année... Cette dérive, que nous constatons actuellement, est aussi le fait de la saturation du marché de l'emploi. Non seulement des étudiants s'inscrivent dans des formations peu regardantes pour pouvoir légalement faire des stages, mais certaines universités proposent des diplômes universitaires (DU), post-master 2, pour alimenter le marché des stages, et ce faisant encaisser des droits d'inscription d'étudiants (qui plus est non limités pour les DU). Nous voyons ainsi des étudiants collectionner les master 2 et les stages dans le secret espoir de parvenir à s'insérer.
La chose ne fait qu'amplifier le phénomène d'une demande croissante de stages et du remplacement des possibilités de vacations par des stagiaires dans les structures.

Seconde conséquence, un stage est véritablement pertinent quand il donne l'occasion à un étudiant de compléter son parcours et d'être un levier dans la construction de son projet professionnel. On ne choisit pas un stage au hasard, parce que l'offre nous est parvenue, parce que l'on doit faire un stage ou parce que papa connaît le conservateur. Ce qui est valable pour un stage d'observation en classe de Troisième ne devrait pas être le réflexe en master pro. Ceci signifie que le stage est pensé en amont par le stagiaire, qu'il est donc argumenté comme une réelle opportunité dans son parcours. Pour cette raison, nous demandons aux étudiants de préciser leur projet avant de choisir un stage et de le faire en fonction de ce que le stage est susceptible de leur apporter. À l'inverse, le lieu de stage devrait s'inquiéter de cette cohérence qui est une garantie d'un stage pleinement réussi.

\section{La nécessaire préparation du stage}

Pour ces raisons, un stage se prépare. Deux documents sont à nos yeux nécessaires pour préparer efficacement un stage en amont. L'un concocté par le lieu de stage, l'autre par le stagiaire : ainsi peuvent se mettre en place les conditions optimales pour que le stage soit efficace et qu'il donne satisfaction à tous.

Le lieu de stage doit mettre sur le papier, souvent pour l'offre de stage, ou dans une sorte de cahier des charges, ce qu'il attend du stage, le contenu de la mission, les conditions et les moyens pour ce faire. C'est une façon pour le lieu de stage de clarifier ses attentes, et ce sera la base du contrat à conclure entre le lieu d'accueil

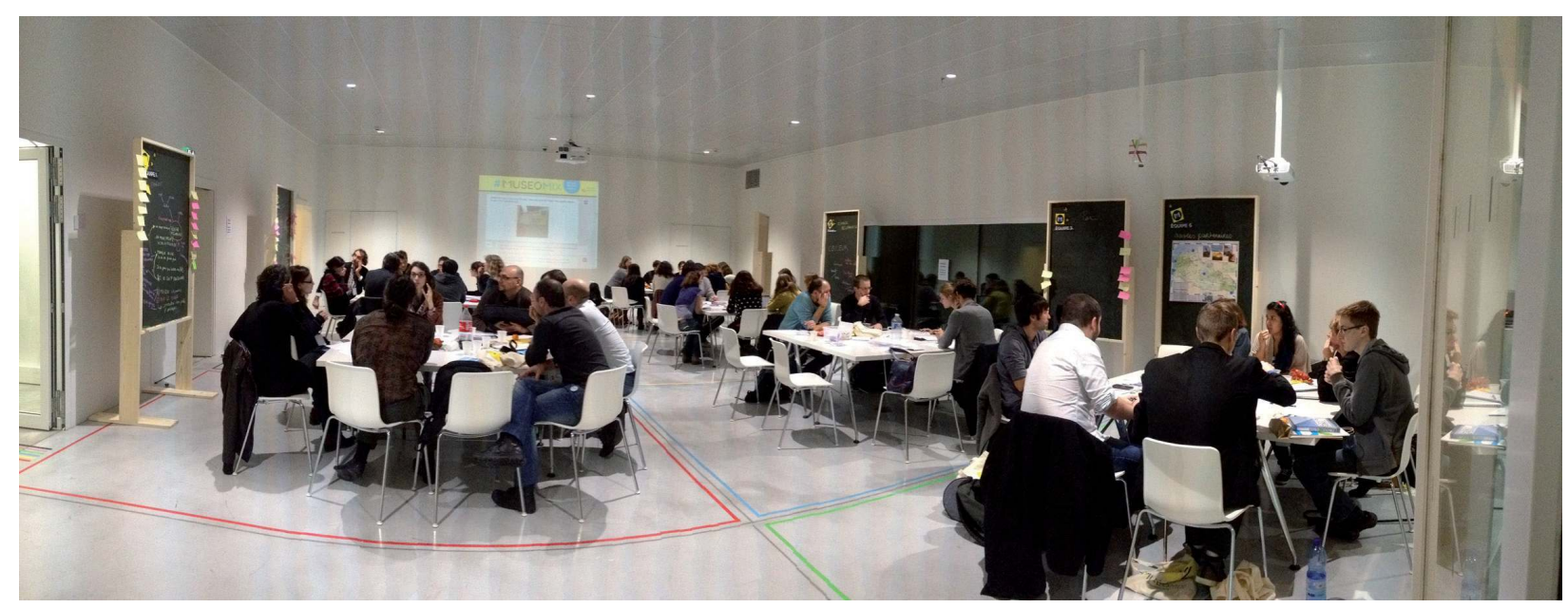

La manifestation Muséomix en Nord, organisée au Louvre-Lens et coordonnée par un groupe projet du master MEM. 
et le postulant. Le document peut détailler plus ou moins la chose, mais une rédaction minimale est nécessaire, garantie contre d'éventuelles dérives postérieures.

Le stagiaire, de son côté, doit préparer une réponse à partir de cette offre. Après avoir candidaté et échangé avec la structure d'accueil, et si les deux parties sont consentantes, le stagiaire doit se préparer à son stage. Les négociations avec les lieux de stage font partie de l'apprentissage à négocier son contrat. C'est pourquoi, à notre sens, le lieu de formation n'a pas à intervenir directement dans cet échange. En revanche, nous demandons aux étudiants de préparer un dossier. Il comprend : une présentation du lieu de stage, de son fonctionnement et de ses actions, si possible de sa gestion et de son organigramme, mais aussi la liste des missions attendues, la méthodologie que l'étudiant va employer pour ce faire, les outils mobilisés, enfin un échéancier de conduite de son travail. Ce dossier remis au tuteur universitaire de la formation sert de document de concertation et d'échange. Lors d'un jury, ou au moins d'un rendez-vous individuel, les responsables de la formation universitaire et le stagiaire peuvent s'en entretenir avant le départ en stage. Pour réaliser ce travail, cela sous-entend que le stagiaire puisse demander au futur lieu de stage des documents bien en amont et que celui-ci lui consacre pour cela un peu d'attention. Ce n'est pas une perte de temps, car plus le stagiaire connaîtra au préalable la structure, plus les missions auront été précisées, plus vite il sera opérationnel au jour J. Ce sont à nos yeux les conditions pour accompagner un stage de manière efficace.

Par ailleurs, une convention est signée, de manière tripartite entre le lieu d'accueil, l'université et le stagiaire définissant les conditions et les périodes de stage pour des questions d'assurance ${ }^{(5)}$. Une annexe à cette convention peut utilement préciser les missions en reprenant la synthèse des documents précédents.

\section{Trouver une place satisfaisante pour chacun}

Durant le stage, il n'est pas rare que les missions évoluent du fait des circonstances, des aléas, des opportunités. Ceci n'est pas problématique, si l'on peut le formaliser en toute connaissance de cause. En revanche, le cahier des charges établi précédemment pourra servir de repères pour s'y reporter en cas de dérives des parties prenantes. Cela permet d'éviter le stage aux promesses extraordinaires qui se réduisent progressivement aux photocopies et à la machine à café ! Cette vision caricaturale n'est pas citée par hasard, mais parce que le stagiaire a été trop souvent mal choisi ou que le stage a été insuffisamment préparé. Préparer un stage est un travail, qui sera d'autant plus satisfaisant qu'on l'aura accompli de part et d'autre avec sérieux.

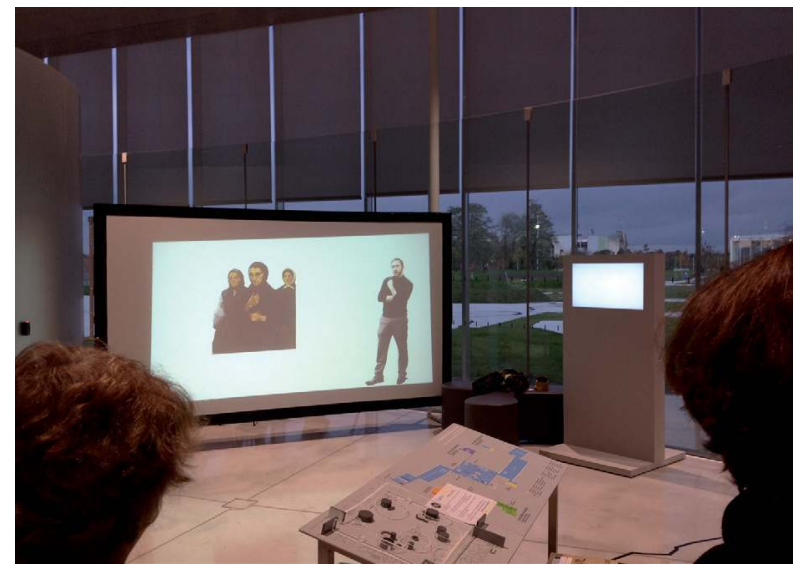

Muséomix en Nord au Louvre-Lens (c) S. Chaumier

Un stagiaire est avant tout dans une situation d'apprentissage. Ceci signifie qu'il n'est pas chargé d'occuper la place d'un salarié, d'un remplacement de congés maternité, d'un emploi saisonnier et encore moins d'un responsable, mais qu'il peut en être l'assistant pour apprendre à ses côtés. Ainsi les missions confiées à un stagiaire doivent être raisonnables et en rapport avec cette situation d'apprentissage. Un étudiant en début de cursus, en licence ou master 1 , se verra confier des missions secondaires, alors qu'un étudiant de master 2 est voué à prendre plus de responsabilité. S'il n'est pas interdit et même recommandé de confier une vraie responsabilité, celle-ci doit être proportionnelle et raisonnable. Nous avons ainsi eu l'occasion de refuser une offre de stage, proposée par un élu, qui demandait un stagiaire pour réaliser le Projet Scientifique et Culturel de son musée. Il faut être logique, si l'on n'a pas de personnes compétentes pour assurer une tâche de cette envergure, comment un stagiaire pourrait-il se former à ses côtés ? Car un stagiaire doit normalement pouvoir se référer à son tuteur pour progresser ou s'il bute sur un problème. Pour cette raison, nous sommes circonspects envers les stages où le stagiaire se retrouve seul, sans référent ou maître de stage dans la place, comme c'est parfois le cas dans nombre de petites structures bénévoles.

Le rôle du tuteur en entreprise est d'accompagner, de donner des avis constructifs pour faire progresser le candidat, non de dénigrer un travail. S'il s'agit de faire refaire, il faut une guidance, qui indique les directions. Rappelons-le, le stagiaire doit être encadré par quelqu'un de compétent. Autre conclusion, on ne peut pas reprocher à un stagiaire de ne pas savoir faire ce que son tuteur n'est pas en mesure de faire lui-même. Si le tuteur n'est pas satisfait du travail accompli, il doit être en mesure d'expliquer pourquoi et de montrer comment il faut le conduire, bref être en mesure de pouvoir l'exécuter. Sans quoi il y a tromperie. 


\section{Définir les missions et accompagner le stagiaire}

Un stage sera d'autant plus satisfaisant qu'il sera un réel exercice d'implication ${ }^{(6)}$. Pour cela, un stage trop court, sauf missions très ponctuelles, n'est pas très gratifiant. Il faut du temps pour s'immerger et conduire un vrai chantier. Selon le niveau académique, le temps est plus ou moins long, mais mieux vaut un stagiaire qui déploie son action sur plusieurs mois que plusieurs stagiaires qui se succèdent, sauf à leur confier des missions dont on peut douter de l'intérêt pour leur apprentissage. Rappelons qu'un stagiaire ne vise pas à remplacer un emploi ne nécessitant pas de qualification. Car a contrario les actions répétitives et purement basiques peuvent être intéressantes sur un temps très court pour un stage de découverte, mais elles ne devraient pas faire l'objet d'un stage long. Par exemple la passation de centaines de questionnaires est pertinente si le stagiaire est véritablement associé en amont et en aval de l'enquête, voire si c'est lui qui en a la responsabilité.

Dans l'idéal, une mission de stage est d'autant plus appréciée qu’elle est clairement énoncée, bien définie et qu'elle donne lieu à un travail visible. Si le travail de coordination est évidemment utile, il est souvent ingrat en cela qu'il supplée à biens des fonctions sans produire un résultat visible. La succession de tâches morcelées, supplétives, peut s'avérer frustrante pour tous. Offrir une mission qui produit un travail identifié comme étant celui réalisé par le stagiaire est évidemment plus gratifiant. Pour éviter toute confusion, il est préférable que le stagiaire se voit confier une mission

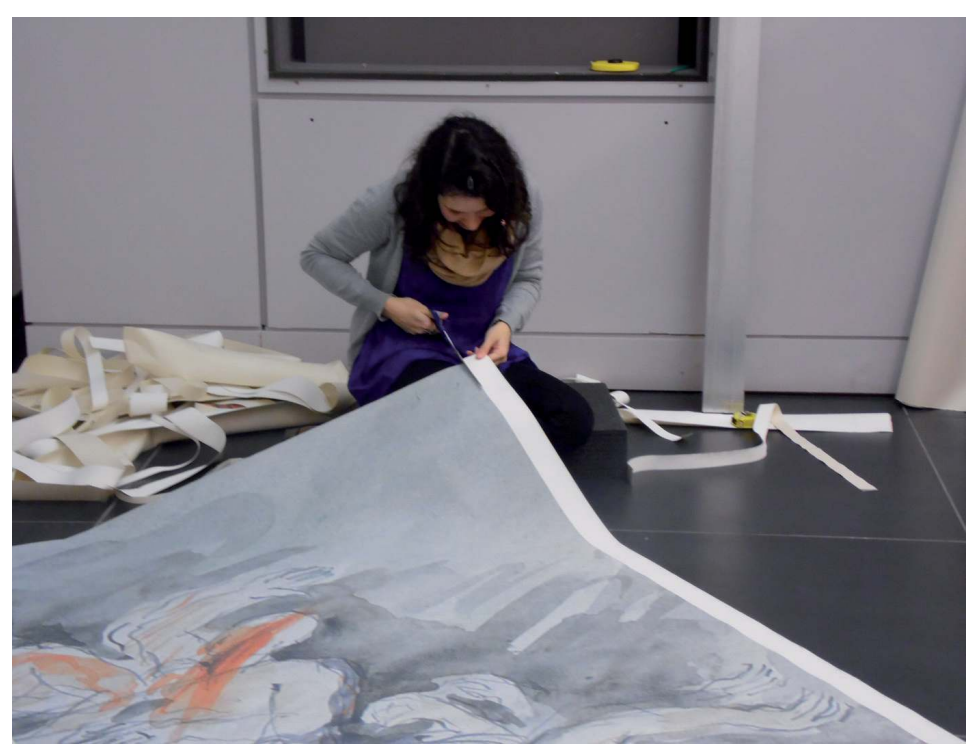

Les stages permettent notamment aux étudiants de participer au montage d'exposition comme ici au musée régional d'Ethnographie de Béthune pour l'exposition Epilepsie, mythes et préjugés. (c) S. Chaumier dont la structure a le projet, mais qu'elle n'a pas le temps d'assumer. Ainsi, le stagiaire apporte un véritable complément, sans prendre la place d'un salarié éventuel. Bien évidemment, il n'y a pas qu'un cas de figure possible et les places occupées par les stagiaires sont nécessairement variables selon les situations et les structures.

Accueillir un stagiaire n'est pas la panacée qui va résoudre tous les problèmes que la structure ne sait gérer elle-même. Si le stage doit être préparé, il convient que le tuteur en entreprise puisse faire confiance au stagiaire à partir de là pour conduire ses missions, mais aussi qu'il veille à faire le point, de temps à autre avec lui. Le temps des études et le temps en entreprise n'est pas le même. Un stage passe vite, fut-il de six mois, période maximale quautorise la loi. Or, dans beaucoup de structures, les mois défilent et les projets s'enchaînent, si bien que les responsables, maladie moderne, sont débordés. Si aux yeux du stagiaire, son stage est crucial dans son parcours, le lieu de stage est pris dans d'autres dynamiques. Savoir consacrer un minimum de temps pour faire le point avec le stagiaire, rectifier certaines incompréhensions ou dérives, est indispensable pour que le partage soit effectif. Accueillir un stagiaire, ce n'est pas seulement bénéficier d'une force de travail à bon compte, c'est savoir y consacrer un minimum d'encadrement. Un stagiaire n'est pas un salarié, il suppose une attention particulière. Ce n'est pas nécessairement lourd en temps, c'est avant tout un état d'esprit dans l'accueil.

Dans la lignée de ce qui précède, prendre le temps de faire le point au terme du stage est le minimum que l'on peut espérer. Savoir évaluer et reconnaître les limites de part et d'autre, n'est pas seulement nécessaire au stagiaire pour avoir un retour sur son expérience, cela peut être utile aussi à la structure pour réfléchir et progresser dans son management. Le stagiaire dispose d'un œil extérieur et pourtant impliqué, candide souvent, mais en cela rafraîchissant pour faire remonter les questions que la structure peut se poser. Même s'il ne faut pas s'illusionner sur la liberté de parole dont il dispose, le stagiaire a malgré tout la possibilité de prendre du recul, une chose qu'un salarié n'a pas nécessairement le temps, l'énergie, l'envie ou le loisir de faire. Le rapport de stage que le lieu de formation demande le plus souvent au stagiaire sert du reste à faire le bilan et à en tirer des enseignements pour l'avenir, d'abord pour lui-même, mais le lieu de stage peut aussi s'en servir comme forme d'analyse.

Si le lieu ne peut attendre trop d'un stagiaire, il ne doit pas non plus en attendre trop peu. Ainsi faut-il se méfier des institutions d'accueil qui déclarent que les stagiaires ne sont que des charges de travail supplémentaire, qu'ils ne savent rien et qu'il faut tout leur 
apprendre. Nous avons ainsi entendu des responsables de structures tenir ce discours méprisant, qui en dit souvent long sur la gestion des ressources humaines dans le lieu et la façon dont souffre l'ensemble des salariés. Car il faut reconnaître que nombre d'organisations disposent d'une gestion chaotique. Un climat détestable en interne rejaillit sur l'accueil des stagiaires. Si ceux-ci sont ainsi immédiatement plongés dans les affres du milieu professionnel, ils sont aussi des analyseurs de cette situation. Il est particulièrement désagréable que ceux-ci en fassent les frais ou soient l'objet de règlements de compte, qu'ils soient boucémissaires ou faire-valoir. Quoiqu'il en soit, les lieux qui sont capables de confier de réelles missions, clairement identifiées, de les encadrer et de faire confiance, sont souvent payés en retour. Ce sont les lieux où les salariés sont eux-mêmes traités de la sorte.

Bien des responsables de stage nous confient leur enthousiasme à l'issue de l'accueil de stagiaire, parce qu'ils sont impressionnés, à partir du moment où le stage

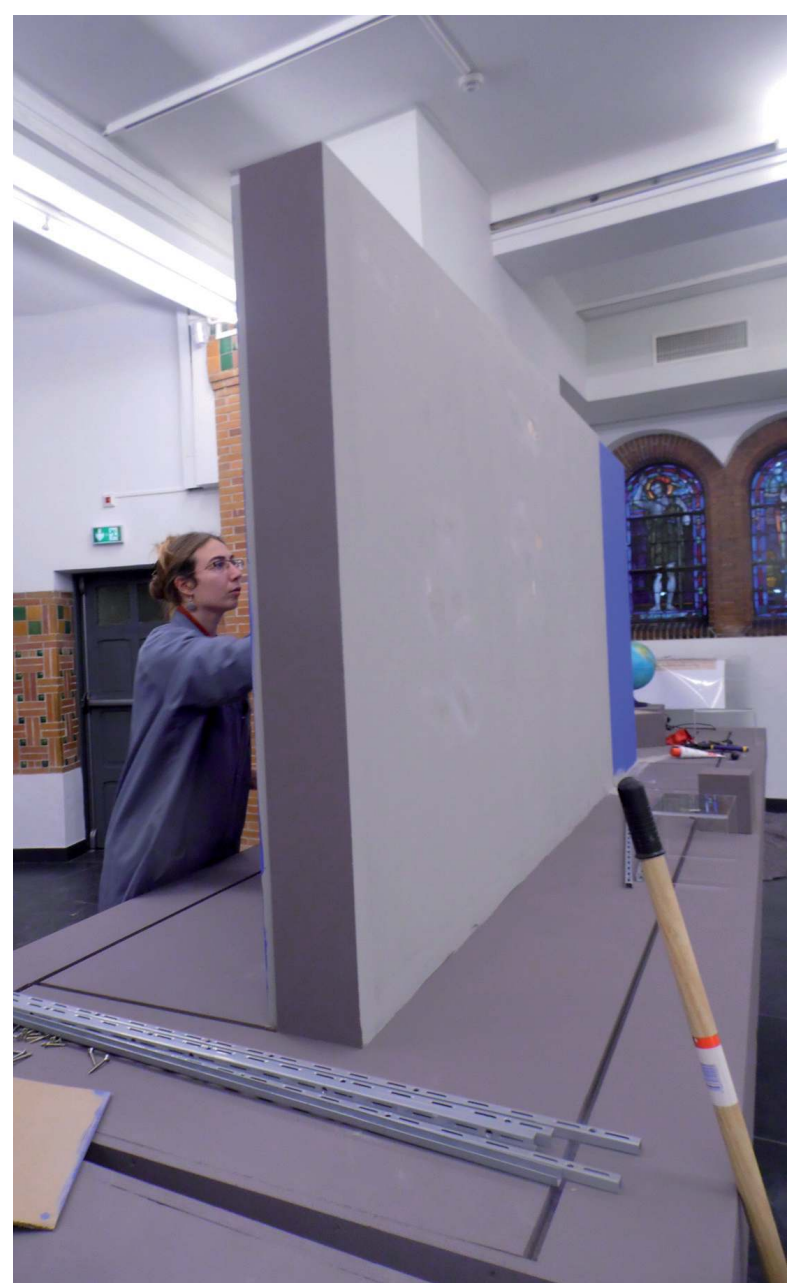

Montage de l'exposition Epilepsie, mythes et préjugés, au musée régional d'Ethnographie de Béthune par une étudiante du master MEM (c) S. Chaumier a été bien préparé et le stagiaire soigneusement choisi en fonction du rôle qu'on lui donne, par l'énergie et l'enthousiasme manifesté par quelqu'un qui entre dans la profession avec la foi et l'élan que n’a plus nécessairement une personne aguerrie. Accompagner un stagiaire, c'est aussi pour l'entreprise d'accueil avoir la possibilité de formaliser, de bénéficier d'une énergie et d'un souffle différent. C'est pour elle, l'occasion d'un rapport d'étonnement. Les métiers de la culture demeurent des métiers de conviction, de passion et de militantisme, c'est en tout cas ainsi que nous les concevons. C'est pourquoi les stagiaires investis sur le terrain, s'ils ont souvent besoin dans un premier temps d'être rassurés sur les compétences qu'ils estiment avec raison ne pas avoir, s'investissent avec d'autant plus de fougue et d'énergie qu'ils doivent faire leurs preuves et d'abord vis-à-vis d'eux-mêmes, mais aussi de l'entreprise, de leur entourage et de leur lieu de formation. L'énergie est donc décuplée dès lors qu'on lui donne les conditions pour s'épanouir. Bien des lieux s'en privent par aveuglement, excès de prudence ou manque de confiance.

\section{Les conditions d'un stage efficient}

Tout travail mérite salaire. En l'occurrence l'adage ici est démenti, puisque le stagiaire fournit bien un travail, mais qu'il ne reçoit pas de salaire. Toutefois des indemnités (de 436,12 euros mensuels) sont désormais obligatoires du moins au-delà de deux mois ${ }^{(7)}$, et toutes les collectivités y seront très certainement astreintes dans un temps assez proche ${ }^{(8)}$. La circulaire adressée aux préfets en date du 25 octobre 2013 en rappelle les conditions. Ce qui importe, c'est l'engagement que cela induit de part et d'autre et la signification symbolique qu'il en ressort. Puisqu'il y a contrat et indemnités, le stage devient d'autant plus sérieux et mérite encadrement formel. Là encore, le fait de dégager des ressources pour indemniser un stagiaire démontre l'importance qu'une structure attache à sa place et au travail attendu. Il est là encore révélateur du peu d'intérêt que manifeste une institution quand elle dédaigne cette gratification ${ }^{(9)}$. Prendre en compte le stagiaire, c'est manifester un intérêt pour ses conditions de vie, ainsi certaines structures proposent des prises en charge en fonction des spécificités du poste (hébergement, frais de déplacements...). La négociation d'un stage passe par ces questions qui expriment là encore la manière dont on envisage dans une structure la gratification des collaborateurs.

Évidemment la reconnaissance passe également par le fait de rendre à César ce qui lui appartient. Un stagiaire sera gratifié si le lieu l'associe aux choix, lui réserve une vraie place, écoute ses propositions et prend en compte ses observations ${ }^{(10)}$. Mentionner le travail 
effectué dans une équipe, dans l'ours d'un générique d'exposition, l'édition d'un ouvrage ou lors du discours d'inauguration n'est pas non plus anodin. La valorisation du stagiaire est aussi une manière de l'accompagner dans sa prise de confiance en lui et dans son affirmation professionnelle. Bien évidemment, les attestations et lettres de recommandations seront des leviers pour aider les stagiaires à aborder leur avenir. Là encore, il ne s'agit pas de beaucoup, et cela semblera évident à bien des lieux d'accueil, mais il n'est pas toujours inutile de le rappeler.

Le lieu d'accueil appréciera que le stagiaire fasse un retour sur son expérience. Et le lieu de formation et ses responsables s'ils ne peuvent visiter tous les stagiaires dans le cadre du lieu de stage, doivent pouvoir être contactés et répondre en cas d'urgence ou de problèmes graves. Ces situations qui arrivent parfois, donnent lieu à concertation et résolution de concert. La visite sur un lieu de stage n'est pas seulement une occasion d'évaluation, c'est surtout l'occasion d'une séance de travail. Cette forme d'échanges peut déboucher sur de nouveaux partenariats. Le rapport de stage est pour le stagiaire une manière de conclure et faire un bilan, et pour les formateurs d'échanger à ce sujet.

Si le rapport de stage peut être demandé par le lieu de stage, en revanche le mémoire que l'étudiant doit parfois effectuer, notamment dans le cadre d'un master 2 , peut ou non être en relation avec le lieu de stage. Parfois le lieu de stage et l'entreprise peuvent être un terrain d'investigation, et les données collectées y sont soumises à autorisation comme pour tous terrains. Toutefois, ce mémoire est un exercice académique, un retour et une problématisation à partir d'une expérience ou d'un terrain d'enquête, il n'est pas inclus dans les missions de stage, pour cela, il regarde exclusivement l'université et l'étudiant qui le conduit. Le lieu de stage peut en demander communication éventuelle, et se le voir ou non communiqué, mais en aucune manière un responsable professionnel n'a à exiger de droit de regard, de validation ou de censure sur ce mémoire. Les choses sont disjointes, c'est un exercice, au même titre que d'autres que la formation conduit avec les personnes en formation.

\section{Le stage, un élément d'un puzzle plus complexe}

Enfin, il faut rappeler combien le stage n'est, en principe, qu'un mode parmi d'autres de la professionnalisation. Il s'accompagne d'autres opérations, et parfois d'autres exercices, comme par exemple des projets tuteurés, des diagnostics ou des réponses à des commandes de la part d'institutions ou d'entreprises. Les occasions de collaborations sont donc nombreuses et diversifiées entre le lieu de formation et les professionnels.

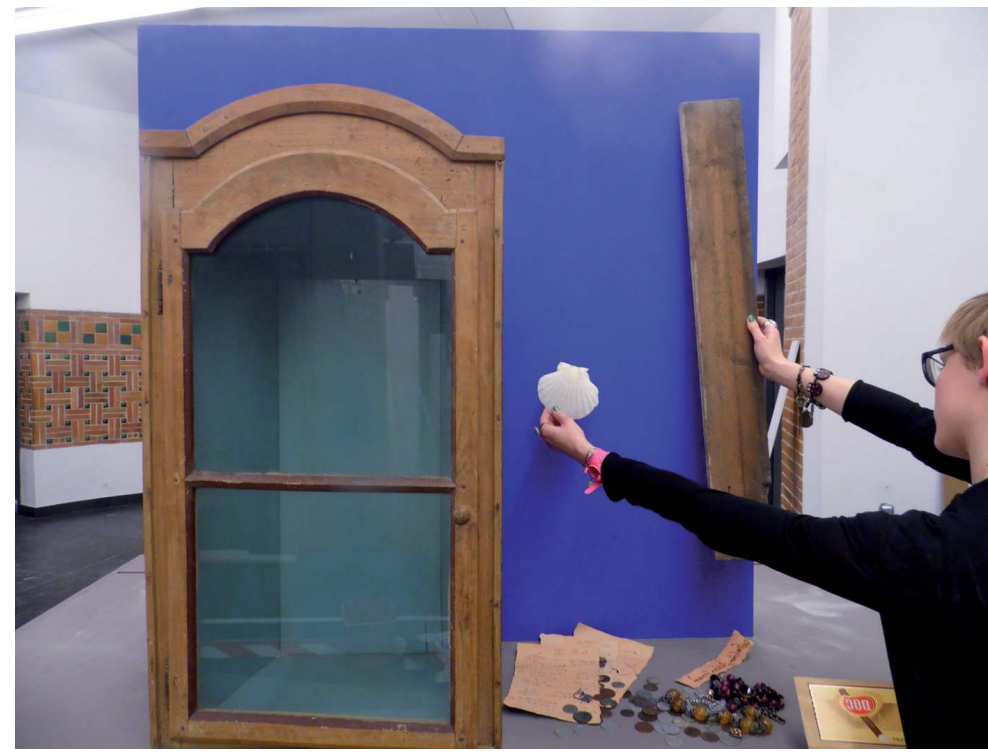

Montage de l'exposition Epilepsie, mythes et préjugés, au musée régional d'Ethnographie de Béthune (C) S. Chaumie

Le stage n'est qu'un maillon pour expérimenter et affiner un projet professionnel, sans devoir enfermer l'étudiant pour l'avenir. Le stage n'est pas la panacée, notamment parce qu'il est limité et parce que trop souvent la relation est distendue, ne serait-ce que par l'éloignement géographique entre lieu d'accueil et lieu de formation. Pour cela, les contrats de professionnalisation ou les contrats d'apprentissage qui offrent aux titulaires de ces contrats de vrais postes de salariés sont plus intéressants pour tous. Pour le bénéficiaire en terme d'expérience et de suivi, d'insertion professionnelle, pour l'entreprise qui bénéficie d'un salarié à moindre coût, présentant plus de fiabilité, sur un plus long terme sans engagement d'embauche, et pour l'université qui peut ainsi développer des relations plus étroites avec les lieux d'accueil. En effet, l'encadrement et le suivi du détenteur d'un contrat de professionnalisation sont beaucoup plus exigeants et induisent un dialogue tout au long du contrat.

Si le stage demeure un outil précieux et essentiel, l'idéal n'est ni dans la multiplication des stagiaires au détriment des salariés dans une entreprise, ni dans la multiplication des expériences de stage à l'infini pour les étudiants. Une inflation, manifeste aujourd'hui, conduit des étudiants à devenir d'éternels stagiaires, les entreprises cherchant parfois au terme du stage à le prolonger en proposant à la personne de devenir de nouveau stagiaire en suivant une xième formation. À la fin d'un stage, si les missions doivent se poursuivre, le recours aux vacations s'impose, et non la pression sur le stagiaire pour qu'il trouve un diplôme où se réinscrire afin de poursuivre son stage l'année suivante... 
Ce détournement de ce qui devrait demeurer un outil en un mode de gestion économique des ressources humaines est évidemment pervers et les associations de défense des stagiaires ont des raisons de protester ${ }^{(11)}$.

Enfin, il faut pour conclure rappeler que si tous les stages n'ont pas pour finalité d'être transformés en emploi, il n'est évidemment pas interdit d'y songer. Rappelons que le chômage des jeunes dépasse désormais les $26 \%$. L'objectif numéro 1 d'une formation professionnelle demeure l'insertion et le stage est l'un des outils pour ce faire, dans la structure, ou ailleurs du fait de l'expérience acquise. L'emploi pour tous demeure l'essentiel, et non le fait de devenir tous stagiaires, selon une formule qui viserait à alléger toujours davantage les charges pesant sur le coût du travail.

Compte tenu de toutes ces remarques et conscients des richesses et des limites qu'offre l'accueil d'un stagiaire, les responsables de formation, les structures d'accueil et les étudiants peuvent partager raisonnablement de belles expériences.

\section{Notes}

(1) Nous remercions nos collègues Isabelle Roussel-Gillet et Daniel Jacobi pour leurs remarques et commentaires sur l'article.

(2) Guillaume Gendron, Depuis 2008, le nombre de stagiaires a doublé, Libération, 9 août 2013. À lire sur www.liberation.fr/societe/2013/08/09/ aujourd-hui-le-stage-phagocyte-tout_923875.
(3) On pourra lire sur ce point l'analyse que nous avions proposée, et dont les travers n'ont fait que s'amplifier depuis : Les formations aux métiers de la culture : de la génération spontanée au désir de régulation, Revue U-Culture, Université de Bourgogne, $\mathrm{n}^{\circ} 1,2006$. Téléchargeable sur mshdijon. u-bourgogne.fr/msh_cnrs/UCultures/Revue_1.pdf

(4) Nous avons consacré un passage à ces questions : Comment choisir une formation ? et Qu'est-ce qu'une bonne formation professionnelle?, dans Chaumier, S. et Mairesse, F. La Médiation culturelle. Armand Colin, 2013, pp. 169-172.

(5) La convention type est disponible sur www.legifrance.fr.

(6) Voir par exemple le témoignage sur : http://lartdemuser.blogspot.fr/ 2013/12/souvenir-de-stage-une-approche-de-la.html

(7) Ce cap des deux mois est du reste actuellement en discussion et sera éventuellement remis en cause par le législateur.

(8) Sylvain Mouillard, Vers une gratification minimale pour tous les stagiaires ?, Libération, 29 mai 2013. À lire sur www.liberation.fr/societe/2013/ 05/29/vers-une-gratification-minimale-pour-tous-les-stagiaires_906630. Du reste, le stage doit désormais être indemnisé, même s'il s'actualise en discontinu dans un même lieu. Si les établissements publics (EPIC) et administrations publiques de l'État sont concernés, les collectivités territoriales n'y sont pas encore astreintes par décret ministériel du 25 octobre 2013, mais le seront probablement prochainement : www.digischool.fr/ vie-etudiante/salaire-stage-reglementation-remuneration-3252.php.

(9) Ainsi ce directeur de centre d'art qui proposait à une postulante stagiaire de nationalité étrangère de convertir l'indemnité en ouvrages invendus, sans apparemment en ressentir grande honte même s'il affichait par ailleurs des postures de contestataire libertaire ! Les nouveaux esclavagistes ne sont pas toujours là où l'on pense...

(10) Stages abusifs : sois stage et tais toi ! Agoravox, www.agoravox.fr/actualites/societe/article/stages-abusifs-sois-stage-et-tais-106215

(11) Voir notamment Générations précaires, www.generation-precaire.org 